\title{
GRAIN AXES COINCIDENCE MODEL: RELAXED CONSTRAINTS AND FINITE STRAINS REVISITED
}

\author{
R. E. BOLMARO
}

\begin{abstract}
Instituto de Física Rosario. CONICET-UNR. Faculty of Science and Engineering-
\end{abstract} Rosario National University. Bv. 27 de febrero 210 bis (2000) Rosario, Argentina

The problem of the kinematics of grain reorientation in polycrystalline plasticity has been addressed many times in the past. A particular grain and the matrix in which that grain is embedded must deform guaranteeing compatibility if no debonding or failure are allowed. This requirement is satisfied by the condition of keeping the main axes of the grain and the hole lodging that grain collinear and of the same size during the whole deformation process. It is not clear yet which one of the many definitions of spin should be considered and how the Taylor model is reproduced for nearly round crystals. The problem is directly connected with spin definitions in continuum mechanics and finite strains. The present paper will be concerned with a rigorous treatment of the many spins and rotations that affect a grain in a polycrystal. It will also be shown that this new treatment suggests a new kind of constraint relaxation that, unfortunately, can not be calculated "a priori" with complete ignorance of the kinetics. However some textures can be simulated. The Los Alamos Polycrystal Plasticity code was modified in order to allow relaxation of some strain components while keeping the reorientations less than a prescribed limit. The resultant textures show the same pattern but different strength than the ones obtained under Taylor assumptions. This Grain Axes Coincidence Model (GACM) has both the Taylor and the Relaxed Constraints and Self Consistent models as natural limits for round and very flat grains respectively. It is shown that the constraint relaxation concept that comes out from the GACM is qualitatively different from the usual in RC and SC models. Strain path changes under the new assumptions are also discussed.

KEY WORDS: Texture, plastic spin, relaxed constraints, polycrystals, finite strains.

\section{INTRODUCTION}

It is by now well known that finite deformation of a suitably small material neighborhood from an arbitrary reference state to another arbitrary state can be viewed as pure stretch along a specific orthogonal triad followed by rigid rotation of the orthogonal triad into another specific orthogonal triad, or, conversely, a rigid rotation followed by pure stretch (Dafalias, 1987; Mehrabadi and Nemat-Nasser, 1987). For convenience, the original state will be called the initial configuration, and the second state will be called the current configuration. There is still another set of axes: the one that remains fixed to the laboratory, sometimes called the background or reference system. The orthogonal triad associated with the material neighborhood in its initial configuration is called the Lagrangean triad and the one associated with the current configuration is the Eulerian triad. This approach, and the consequent definition of polar decomposition, leads to simple relations among various strain measures, deformation rates and spin measures. 
Let $d \mathbf{X}=d X_{j} \mathbf{e}_{\mathbf{j}}$ be an elementary material line element in the reference configuration $C_{o}$. The mapping that deforms this line element into $d \mathbf{x}=d x_{i} \mathbf{e}_{\mathrm{i}}$ is given by

$$
\mathbf{d x}=\mathbf{F d X},
$$

where $\mathbf{F}=\partial \mathbf{x} / \partial \mathbf{X}$ is the deformation gradient with components:

$$
F_{i j}=x_{i, \cdot}
$$

The transformation (1.1) rotates and stretches material lines, and therefore, a material neighborhood is, in general, rotated as well as distorted. To uncouple the rigid rotation from the pure distortion, the polar decomposition of $F$ is used:

$$
\mathbf{F}=\mathbf{R U}=\mathrm{VR}, \quad F_{i j}=R_{i k} U_{k j}=V_{i l} R_{l j}
$$

where the symmetric positive-definite tensors $U$ and $V$ are called the right and left stretch tensors, and $\mathbf{R}$ is a proper orthogonal matrix such that

$$
\mathbf{R}^{-1}=\mathbf{R}^{\mathbf{T}}, \quad \operatorname{det} \mathbf{R}=+1 .
$$

It has been proved that this polar decomposition is unique.

It can also easily be proved that

$$
\mathbf{C}=\mathbf{F}^{\mathrm{T}} \mathbf{F}=(\mathbf{R U})^{\mathbf{T}}(\mathbf{R U})=\mathbf{U}^{2}=(\mathbf{V R})^{\mathbf{T}}(\mathbf{V R})=\mathbf{R}^{\mathrm{T}} \mathbf{V}^{2} \mathbf{R}=\mathbf{R}^{\mathrm{T}} \mathbf{B} \mathbf{R}
$$

where

$$
B=V^{2}=F^{T}=R^{T} R^{T} .
$$

The quantity $\mathbf{B}$ is called the left Cauchy-Green tensor.

At each material point the mapping $d \mathbf{x}=\mathbf{F} d \mathbf{X}$, characterized by the deformation gradient $\mathbf{F}$, identifies two unit orthogonal triads: one consisting of the unit vectors $\mathbf{N}^{(\mathbf{i})}$ which are the principal directions of the right stretch tensor $\mathbf{U}$, called the Lagrangean triad, and the other consisting of the unit vectors $\mathbf{n}^{(\mathbf{i})}$ which are the principal directions of the left stretch tensor $\mathbf{V}$, called the Eulerian triad. The rotation $\mathbf{R}$ maps the Lagrangean into the Eulerian triad.

The rotation of the $\mathbf{e}_{(\mathbf{j})}$-triad to the Eulerian $\mathbf{n}^{(\mathbf{i})}$-triad, $\mathbf{R}^{\mathbf{E}}$, then may be viewed as the rotation of the $\mathbf{e}_{(j)}$-triad to the Lagrangean $\mathbf{N}^{(\mathbf{j})}$-triad, $\mathbf{R}^{\mathbf{L}}$, followed by the rotation of the $\mathbf{N}^{(\mathbf{j})}$-triad to the $\mathbf{n}^{(\mathbf{i}) \text {-triad, }} \mathbf{R}$. Hence

$$
\mathbf{R}^{\mathbf{E}}=\mathbf{R R}^{\mathbf{L}} \text {. }
$$

Another useful quantity is the velocity gradient

$$
d \mathbf{v}=\left(\partial \mathbf{v} / \partial x_{j}\right) d x_{j}=\mathbf{L} d \mathbf{x},
$$

which can be written

$$
\mathbf{L}=\dot{\mathbf{F F}}^{\mathbf{- 1}} \text {. }
$$

The velocity gradient is decomposed into the symmetric part, $\mathbf{D}$, called the deformation rate tensor, and the antisymmetric part, $\mathbf{W}$, called the spin tensor, the twirl tensor or vorticity:

$$
\begin{array}{ll}
\mathbf{L}=\mathbf{D}+\mathbf{W} & \\
\mathbf{D}=1 / 2\left(\mathbf{L}+\mathbf{L}^{\mathbf{T}}\right), & d_{i j}=1 / 2\left(l_{i, j}+l_{j, i}\right) \\
\mathbf{W}=1 / 2\left(\mathbf{L}-\mathbf{L}^{\mathbf{T}}\right), & w_{i j}=1 / 2\left(l_{i, j}-l_{j, i}\right)
\end{array}
$$


In these equations $\mathbf{D}$ characterizes the instantaneous rate of deformation, and $\mathbf{W}$ the instantaneous rate of rotation of the material neighborhood. The vorticity $\mathbf{W}$ has been proved to be the average spin of the material lines that go through the center of spinning (Cauchy, 1841).

In the course of deformation, both the Lagrangean and the Eulerian triads, $\mathbf{N}^{(i)}$ and $\mathbf{n}^{(j)}, i, j=1,2,3$, continually change. Let $\Omega^{\mathbf{L}}$ and $\Omega^{\mathbf{E}}$ be the corresponding spin tensors such that

$$
\begin{aligned}
& \dot{\mathbf{R}}^{\mathbf{L}}=\Omega^{\mathbf{L}} \mathbf{R}^{\mathbf{L}} \\
& \dot{\mathbf{R}}^{\mathbf{E}}=\boldsymbol{\Omega}^{\mathbf{E}} \mathbf{R}^{\mathbf{E}}
\end{aligned}
$$

and let the spin of the Lagrangean triad relative to the Eulerian triad be denoted by $\mathbf{\Omega}^{\mathbf{R}}$ :

$$
\dot{\mathbf{R}}=\Omega^{\mathbf{R}} \mathbf{R} \text {. }
$$

If we write the components of tensors $U, \dot{U}$ and $\Omega^{L}$ on the Lagrangean triad, say, $u_{i j}, \dot{u}_{i j}, \Omega^{L}{ }_{i j}$, and the components of tensors $\mathrm{D}, \mathbf{W}, \Omega^{\mathbf{E}}$ and $\Omega^{\mathrm{R}}$ on the Eulerian triad, say, $d_{i j}, w_{i j}, \Omega_{i j}{ }_{i j}, \Omega^{R}{ }_{i j}$, then the following relations can be found for the defined spins:

$$
\begin{array}{ll}
\Omega_{i j}^{R}=w_{i j}-\frac{\lambda_{i}-\lambda_{j}}{\lambda_{i}+\lambda_{j}} d_{i j} & \text { (no sum) } \quad\left(\lambda_{i} \neq \lambda_{j}\right) \\
\Omega_{i j}^{E}=w_{i j}-\frac{\lambda_{i}^{2}-\lambda_{j}^{2}}{\lambda_{i}^{2}+\lambda_{j}^{2}} d_{i j} & \text { (no sum) } \quad\left(\lambda_{i} \neq \lambda_{j}\right) \\
\Omega_{i j}^{L}=-\frac{2 \lambda_{i} \lambda_{j}}{\lambda_{i}^{2}-\lambda_{j}^{2}} d_{i j} & \text { (no sum) } \quad\left(\lambda_{i} \neq \lambda_{j}\right)
\end{array}
$$

where the $\lambda_{j}-s$ are the eigenvalues of $\mathbf{U}$ and $\mathbf{V}$.

The rotations and spins can also be written as

$$
\begin{aligned}
& \mathbf{R}^{\mathbf{L}}=\mathbf{N}^{(\mathbf{i})} \otimes \mathbf{e}_{(\mathbf{i})} \\
& \mathbf{R}^{\mathbf{E}}=\mathbf{n}^{(\mathbf{i})} \otimes \mathbf{e}_{(\mathbf{i})} \\
& \mathbf{R}=\mathbf{n}^{(\mathbf{i})} \otimes \mathbf{N}_{(\mathbf{i})} \\
& \mathbf{\Omega}^{\mathbf{L}}=\Omega_{i j}^{L} \mathbf{N}^{(\mathbf{i})} \otimes \mathbf{N}^{(\mathbf{j})} \\
& \mathbf{\Omega}^{\mathbf{E}}=\Omega_{i j}^{E} \mathbf{n}^{(\mathbf{i})} \otimes \mathbf{n}^{(\mathbf{j})} \\
& \mathbf{\Omega}^{\mathbf{R}}=\Omega_{i j}^{R} \mathbf{n}^{(\mathbf{i})} \otimes \mathbf{n}^{(\mathbf{j})}
\end{aligned}
$$

Another spin definition is the spin of the main strain rate axes. It will be denoted by $\Omega^{\mathbf{D}}$ and it has to be distinguished from $W$ in the sense that $\Omega^{\mathbf{D}}$ is the spin of an orthogonal set of axes (Bolmaro and Kocks, 1992; Guo et al., 1992) meanwhile W is the average spin of the infinite set of lines that goes through the spinning center (Cauchy, 1841). 


\section{GRAIN REORIENTATION AND GRAIN AXES SPIN FOR FLAT GRAINS}

In the Kroner-Taylor-Bishop and Hill formulation the total (macroscopic) distortion tensor $\mathbf{L}$ (velocity gradient) is taken as uniform throughout the polycrystal; the plastic strain tensor being also taken as uniform, the lattice rotation can be deduced by the differential equation:

$$
w_{i j}^{*}=w_{i j}-w_{i j}^{P}\left(\mathbf{D}, \mathbf{R}^{*}\right),
$$

where $w_{i j}$ is the antisymmetric part of $\mathbf{L}$ and

$$
\left.w_{i j}^{P}=\left(b_{i}^{s} n_{j}^{s}-b_{j}^{s} n_{i}^{s}\right) \dot{\gamma}^{s} / 2 . \quad \text { (sum over } s\right)
$$

In eqn. (2.2), $\mathbf{s}$ is the index of the slip system, $\mathbf{n}^{\mathbf{s}}$ and $\mathbf{b}^{\mathbf{s}}$ are the normal to the slip plane and slip direction, respectively, and $\dot{\gamma}^{s}$ is the microscopic shear strain rate on that system. This "plastic rotation" is function of the current $\mathbf{D}$ and current orientation of the grain lattice $\mathbf{R}^{*}$.

When grains become nonequiaxed, all components of the total distortion tensor may not be transmitted from grain to grain and an appropriate calculation has to be done considering the grain shape. The equation (2.1) is still taken as valid locally with the local tensor $w_{i j}$ now being different from the average total antisymmetric part of $\mathbf{L}$. Many mechanisms and models have been proposed for calculating the local effect of grain flatness. An extensive discussion of the Relaxed Constraints (RC) model can be found in Kocks and Chandra (1982). Self consistent schemes also allow for the grains to have strains different from the surrounding matrix (Molinari et al., 1987). Kocks et al. (1992) tried to generalize the RC model by using the equation (1.17) and interpreting the mismatch of the hole and deforming grain as the cause for the departure of the crystallographic spin from the usual equation (2.1).

The tensor $\mathbf{R}^{\mathbf{E}}$ represents the orientation of the grains's Eulerian axes. The orientation of those grain axes can be reckoned, step by step, as the eigenvectors of $\mathbf{B}$; and their lengths, by the square roots of the eigenvalues of $\mathbf{B}$. The whole idea states that a piece of polycrystalline material can be characterized by its texture and by the orientation of the grain ellipsoids. Even though the model has not been stated as it follows by Kocks et al. (1992), it can be understood in the following alternative reinterpretation.

The equation (1.17) is the key equation for the argument. If we assign an ellipsoid to the grain and another one to the hole where it is located (and it should remain), we can calculate both Eulerian spins. The hole Eulerian spin is simply the one calculated with the macroscopic $w_{i j}, d_{i j}$ and $\lambda_{i}-s$.

$$
\Omega_{i j}^{E_{m}}=w_{i j}^{m}-\frac{\lambda_{i}^{2}+\lambda_{j}^{2}}{\lambda_{i}^{2}-\lambda_{j}^{2}} d_{i j}^{m} \quad \text { (no sum) } \quad\left(\lambda_{i} \neq \lambda_{j}\right)
$$

where $m$ stands for macroscopic.

The grain Eulerian spin is reckoned through the slip system activity necessary for accomplishing the strain under the particular model we are dealing with.

$$
\Omega_{i j}^{E_{g}}=w_{i j}^{g}-\frac{\lambda_{i}^{2}+\lambda_{j}^{2}}{\lambda_{i}^{2}-\lambda_{j}^{2}} d_{i j^{\circ}}^{g} \quad \text { (no sum) } \quad\left(\lambda_{i} \neq \lambda_{j}\right)
$$


If we assume that both sets of principal axes should remain collinear in order to guaranteeing compatibility, the "extra spin" appears as the difference between both (2.3) and (2.4). The $\lambda_{i}-s$ should be the same for both the macro and microscopic level because the grain must fit the hole not only in direction but also in size. Then, calling the combination of $\lambda_{i}-s$ as $\beta_{i j}$ :

$$
\Omega_{i j}^{*}=\Omega_{i j}^{E_{m}}-\Omega_{i j}^{E_{g}}=w_{i j}^{m}-w_{i j}^{g}\left(\mathbf{D}, \mathbf{R}^{*}\right)-\beta_{i j}\left(d_{i j}^{m}-d_{i j}^{8}\right) \quad \text { (no sum) }
$$

which is very close to (2.1) except for the last term $\left(w^{m}{ }_{i j}\right.$ is $w_{i j}$ and $w^{g}{ }_{i j}$ stems from the pure plastic glide and is identical to $\left.w^{p}{ }_{i j}\right)$. The spin $w^{*}$ has been substituted by $\Omega_{i j}^{*}$, meaning that it is the kind that can be written as $\mathbf{R}^{*} \mathbf{R}^{*} \mathbf{T}$, where $\mathbf{R}^{*}$ is the orientation of a set of axes (crystal axes in this case), and not the kind that represents an average spin of a bunch of orientations as $w_{i j}$ does.

Different polycrystal models make different assumptions about the possible inhomogeneity of deformation. Whenever we applied RC models $d_{i j}^{g}=d^{\mathrm{m}}{ }_{i j}$ on the very first steps and some of them become different at higher deformations. That would make (2.5) exactly like (2.1) on the first steps and would reproduce the usual RC expressions for later steps $\left(\beta_{i j}=1\right)$. Unfortunately $\beta_{i j}$ diverge when the aspect ratio of the grains is one. That makes the last term impossible to calculate, if not divergent by itself, It can be applied just after a few percentage of deformation, when $\beta_{i j}$ is close enough to 1 but it is still approaching to one from values larger than one. This model also needs the introduction of external hypothesis in order to calculate the strain relaxation. The antisymmetrical part $w^{g}{ }_{i j}$ is consequence of the slip systems activated under the special RC assumptions and can not be modified independently.

Equations (2.1) and (2.5) are nothing more than the differences between the macroscopic and crystal skew symmetric parts of the velocity gradient or the spin of Eulerian axes, respectively. It will be shown that an alternative equation involving reorientation of Lagrangean axes comes out from the polar decomposition.

\section{NEW SPIN SCHEME}

Simply put, the extra spin is the difference of the rate of rotation (spin) of the substructure (crystal lattice spin) from the rate of rotation of the continuum (material spin). This will be obtained by considering the whole deformation process composed of two different ones. The material deformation gradient is represented by the polar decomposition given by eqn. (1.3). Shortly speaking, it is the deformation gradient of the grain neighborhood, that is to say the deformation gradient affecting the hole in which the grain is lodged.

Meanwhile, the deformation gradient affecting the grain itself can be divided in two different and subsequently applied operators as seen in Figure 1: a) plastic deformation and b) elastic deformation plus 'any other cause for rotation'.

$$
\mathbf{F}=\mathbf{F}^{*} \mathbf{F}^{\mathbf{P}}=\mathbf{R}^{*} \mathbf{U}^{*} \mathbf{R}^{\mathbf{P}} \mathbf{U}^{\mathbf{P}}
$$

where

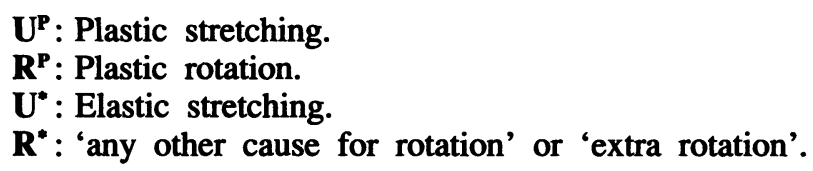




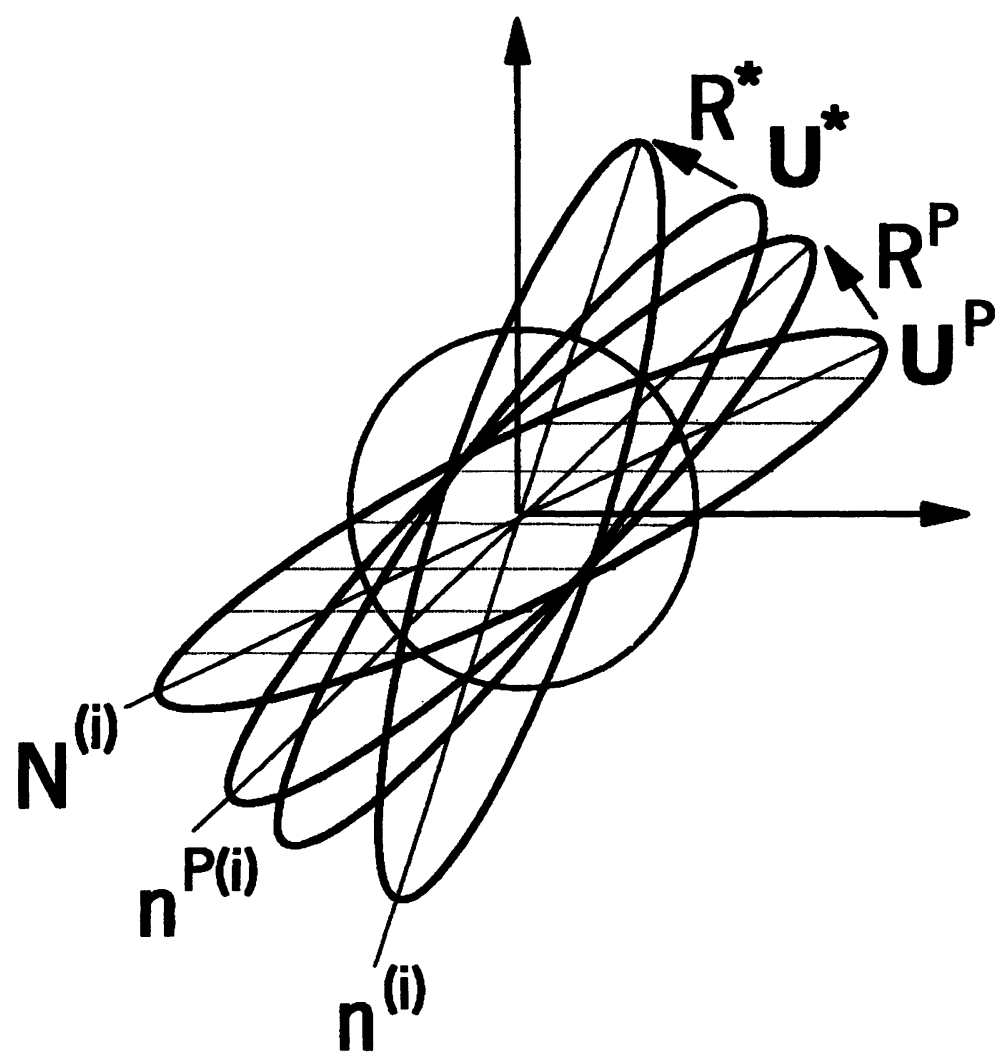

Figure 1 Polar decomposition of the grain deformation gradient.

$\mathbf{R}^{*}$ has been called the 'elastic rotation' but it is more than that in two senses: The magnitude of this rotation exceeds largely the usual elastic distortions and it is not the kind of recoverable distortions that can be considered elastic. This decomposition introduces a new intermediate unit orthogonal triad $\mathbf{n}^{\mathbf{P}(i)}$ that is related with the

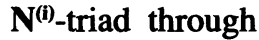

$$
\mathbf{n}^{\mathbf{P}()}=\mathbf{R}^{\mathbf{P}} \mathbf{N}^{(\mathbf{)}}
$$

The elastic stretching can be considered nil compared with the large plastic deformations involved. Eqn. (3.1) is reduced to

$$
\mathbf{F}=\mathbf{R}^{*} \mathbf{R}^{\mathbf{P}} \mathbf{U}^{\mathbf{P}} \text {. }
$$

The macroscopic or hole deformation gradient and the grain deformation gradient must be identical. That condition yields:

$$
\mathbf{F}=\mathbf{R}^{*} \mathbf{R}^{\mathbf{P}} \mathbf{U}^{\mathbf{P}}=\mathbf{R} \mathbf{U}
$$

Both deformation gradients being equal the polar decomposition is unique and the following identities must separately be fulfilled:

$$
\begin{gathered}
\mathbf{R}=\mathbf{R}^{\mathbf{*}} \mathbf{R}^{\mathbf{P}} \\
\mathbf{U}=\mathbf{U}^{\mathbf{P}} .
\end{gathered}
$$


$\mathbf{R}$ can be differentiated by the rule of chain

$$
\dot{\mathbf{R}}=\dot{\mathbf{R}}^{*} \mathbf{R}^{\mathbf{P}}+\mathbf{R}^{*} \dot{\mathbf{R}}^{\mathbf{P}}
$$

and also transposed

$$
\mathbf{R}^{\mathbf{T}}=\mathbf{R}^{\mathbf{P T}} \mathbf{R}^{* \mathbf{T}}
$$

what yields

$$
\begin{aligned}
& \dot{\mathbf{R}} \mathbf{R}^{\mathbf{T}}=\dot{\mathbf{R}}^{*} \mathbf{R}^{* \mathbf{T}}+\mathbf{R}^{*} \dot{\mathbf{R}}^{\mathrm{P}} \mathbf{R}^{\mathrm{PT}} \mathbf{R}^{* \mathbf{T}} \\
& \mathbf{\Omega}=\mathbf{\Omega}^{*}+\mathbf{R}^{*} \mathbf{\Omega}^{\mathbf{P}} \mathbf{R}^{*} \mathbf{T} .
\end{aligned}
$$

This expression can be written component by component as

$$
\Omega_{i j}=\Omega_{i j}^{*}+\Omega_{i j}^{p}
$$

with the first member and the first term in the second member expressed in the Eulerian axes $\mathbf{n}^{(\mathbf{i})}$ and the second therm in the second member expressed in the intermediate plastic triad $\mathbf{n}^{\mathbf{P}(\mathbf{i})}$.

The eqn. (3.10) can be written in any set of axes. If the extra rotation $\mathbf{R}^{*}$ is close to identity, what is true for calculation steps small enough, the $\mathbf{n}^{\mathbf{P ( i )}}$-triad will be closer to the Eulerian triad and $\Omega^{*}$ is calculated as the difference of the plastic spin $\Omega^{p}$ from the macroscopic spin $\Omega$ written in Eulerian axes:

$$
\Omega^{*}=\Omega-\Omega^{\mathbf{P}}
$$

By doing that both terms in the second member can be decomposed in two terms each in agreement with equation (1.16). The symmetrical and antisymmetrical tensors used in the decomposition of $\Omega^{\mathrm{P}}$ stem from the pure plastic crystallographic slip. The $\lambda_{i}-s$ involved in both terms can be considered identical because the grain and the hole must have the same shape and size during the whole process. Thus, $w_{i j}, d_{i j}, w^{P_{i j}}$, and $d_{i j}^{P}$ have self-explanatory meanings.

Rearranging and replacing the function of $\lambda_{i}-s$ by

$$
\alpha_{i j}=\frac{\lambda_{i}-\lambda_{j}}{\lambda_{i}+\lambda_{j}}
$$

it leads to

$$
\Omega_{i j}^{*}=w_{i j}-w_{i j}^{p}-\alpha_{i j}\left(d_{i j}-d_{i j}^{p}\right) . \quad \text { (no sum) }
$$

This equation has several characteristiques to address: a) The first term is something we do not know 'a priori' and that we want to calculate in Eulerian axes or current set of axes. b) The magnitudes $w_{i j}$ and $d_{i j}$ are magnitudes we know either from the boundary conditions or from the particular strain-stress field we are considering. c) The magnitudes involved are usually known in a set of Eulerian axes in a former calculation step. The new Eulerian set of axes is still to be known at the current calculation step but, with calculation steps small enough, there should be not too much error introduced by using the values in the foregoing Eulerian triad. Actually $\Omega^{P}$ should be written in the intermediate $\mathbf{n}^{\mathbf{P}(i)}$-triad but again, for computational purposes, the components in the precedent Eulerian triad will be close enough to the current ones.

Comparing with the results of Tiem et al. (1986) we find that the factors $\alpha_{i j}$ have the right variation and they never diverge thus allowing a continuous calculation. The 
last term will be nil on the first stages of deformation, no matter how important could be the difference between both strain rates, if we start from round grains. It will become more and more important as the deformation proceeds. This equation shows no divergence for round particles and it is more forgiveness in this case because, no matter how different the grain and the macroscopic strains are, they are allowed to proceed because the $\alpha_{i j}$ parameters are nil at this stage.

Unfortunately we still have the problem of calculating which strain rate is relaxing and how much. We can go through the kinetics of the problem and solve it with a scheme similar to the usual RC models or we can calculate the grain strain rate under a Self Consistent scheme. Further interpretations will be given later.

The basic assumption is that each crystal will modify its internal strain rates and consequent spins in order to achieve the conditions of equal orientation of grain axes and equal eigenvalues. That means that the grain will choose slightly different strain rate paths in order to achieve the right rotation to keep the grain and hole main axes collinear. We have to keep in mind that the reorientation cannot be accomplished without strong inhomogeneities inside the grain if we do not allow the grain boundary to slide freely (Zolotorevskiy et al., 1989. Harren and Asaro, 1989). There are however some experimental evidences in support of the opposite result. The grains in a single phase polycrystal material show almost the same deformation as the macroscopic one if it is characterized by shape, size and orientation of the associated ellipsoids. High fragmentation or internal inhomogeneities are not observed in the most of the tests and materials (Kurzydlowski et al., 1992). Therefore there will be local stress evolution, while the grain will try to deform with the closest spin to the macroscopic one $\left(\Omega^{*}{ }_{i j}=0\right)$ in order to preserve: a) the integrity of the grain, homogeneity and no fragmentation and b) matching of hole and grain shape and size. If the stress evolution is successful in developing different strain components and accomplishing $\Omega^{*}{ }_{i j}=0$ there will be no extra spinning. When that is not possible, as in very anisotropic and inhomogeneous materials, the grains will fragment, deform inhomogeneously and spin consequently with this behavior. It seems as if crystal axes spin, other than the induced by macroscopic average spin, is always related with grain inhomogeneities. Actually $\boldsymbol{\Omega}^{*}{ }_{i j}=0$ never happens because inhomogeneities do exist. Twinning would be a way of fragmenting for anisotropic materials even though fragmenting and debonding are usually energetically unfavorable.

Let us discuss the span of the relaxation concept for this model. The equation (3.14) shows that shear strain rates (in grain axes) can be accommodated to accomplish grain and hole matching by simultaneous modification of a related longitudinal strain rate. In a pure shear test, where the axis of compression is the Z-axis and the expansion is along the $\mathrm{Y}$-axis, the most important foreseen relaxed shear strain rate component is $d_{13}$. But also $d_{23}$ and $d_{12}$ will relax. Moreover, the modification of any shear strain rate should modify the associated diagonal components. The relaxation of $d_{13}$ must modify $d_{11}$ and $d_{33} ; d_{23}$ relaxation has to change $d_{22}$ and $d_{33}$ and so on. We will introduce the 'ad hoc' hypothesis of keeping the same equivalent Von Mises strain step by step and grain by grain. The same phenomena of associated relaxation happens for a simple shear test where the spin is around the $\mathrm{X}$-axis. This makes a big difference with the usual RC and SC models. This kind of local modification of strain rates is a qualitatively different one. In the RC and SC models the different components will relax allowed by strain inhomogeneities developed in the grain boundary. In the current model some components are relaxing because the grains are not allowed to develop inhomogeneities. In the SC models the relaxing strain components are compensated in average by the 
rest of the grains. In the current model they are internally compensated grain by grain just by keeping constant the Von Mises equivalent deformation. In this case the relaxation can be quite important. This is a sort of generalized Taylor hypothesis (or; better to say, a collapsed Taylor hypothesis) in the sense that the strains and strain rates must be kept constant locally in the criteria of Von Mises deformation and not component by component. Self consistent approaches usually find a 10-20\% standard deviation in strain rate but it is still to be known if this deviation is kept in the same order for each grain along the whole deformation process. For the time being there is no experimental evidence for preferential apportion of the strain to some particular grains (Kurzydlowski et al., 1992). Self consistent simulations are planned to find out whether the time average deformation for each grain equals the average macroscopic deformation or not (Lebensohn and Bolmaro, 1993).

What should we expect from a model like this one? We can predict a higher spreading of orientations or smoothing of pole figures. They will not be as strong as in previous Taylor based simulations. The Los Alamos polycrystal plasticity (LApp) code (Los Alamos Polycrystal Plasticity code, 1988) is designed to simulate test cases such as proportional loading under various fixed boundary conditions. We found spins higher than $\mathbf{0 . 0 4}$ for velocity gradients with strain rates equivalent to a Von Mises strain of 0.025 per step (Bolmaro and Kocks, 1992). They seem to be too high in order to guarantee the integrity of the polycrystal and low inhomogeneities. We have slightly modify the LApp code in order to allow strain rates relaxation simultaneously keeping the crystal axes spin below an arbitrarily fixed limit. A Newton-Raphson method was implemented to keep the spin calculated by eqn. (3.14) below that fixed limit. The textures obtained are weaker than the Taylor based simulations but showing the same pattern. Figure 2 a) shows the [111] pole figure for torsion of an FCC material at a Von Mises equivalent strain of 2.00 simulated under Taylor assumption and no limit for the crystal axes spin. The intensities come out stronger than the usual experimental textures. Figure 2 b) shows the same simulation but keeping the crystal axes spin bellow 0.03 per strain step of 0.025 Von Mises equivalent strain. The texture is weaker than the one shown in Figure 2 a) and it captures better the decrement of the B fiber on benefit of the $A$ fiber. This a result that can be observed experimentally and can be explained with the assumptions of the present model.

This model is still lacking of a kinetical law for $\left(d_{i j}-d_{i j}{ }_{i j}\right)$ RC or Self-Consistent approaches are good candidates as they were in the past (Lebensohn and Bolmaro, 1993) to model strain compensation among different grains. Although when the standard deviation of strains and stresses are higher than 10-20\% respect to the average a onesite SC approach should be replaced by a higher order one. Moreover, the problem of internal compensation might not be considered by the classical one-site implementations but for descriptions taking in account the detailed topology.

\section{SOMETHING ABOUT STRAIN PATH CHANGES}

The concept of strain path change has been discussed by Takeshita et al. (1988). Dresselhauss and Tabor (1992) found a parameter to describe the alignment of a material element with the principal axes of strain in fluid mechanics that is identical to the one obtained by Bolmaro and Kocks (1992) to characterize strain path changes in texture development. In fluids it is supposed that, like in Taylor approach, there is no shape interaction between the material element and the surroundings. In this sense the concept 


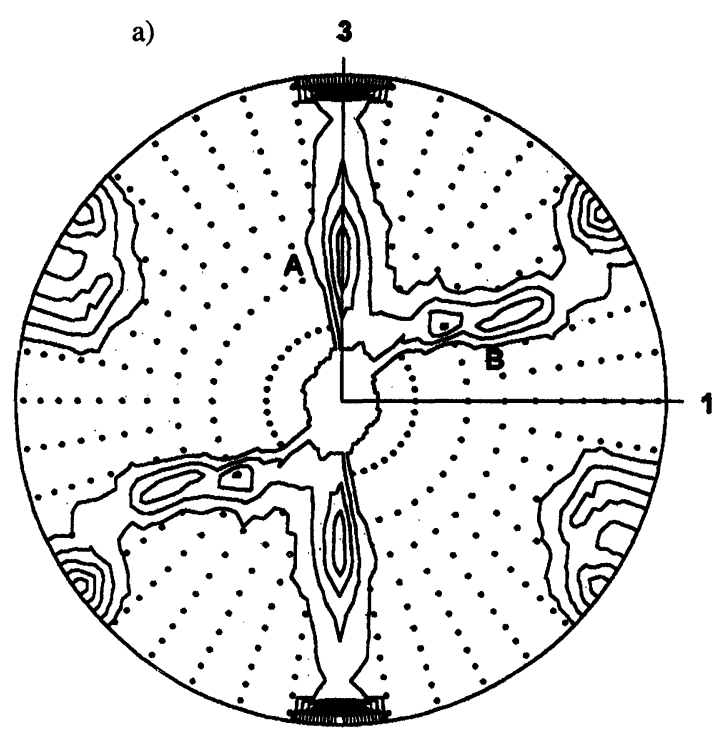

\section{[111] pole figure}

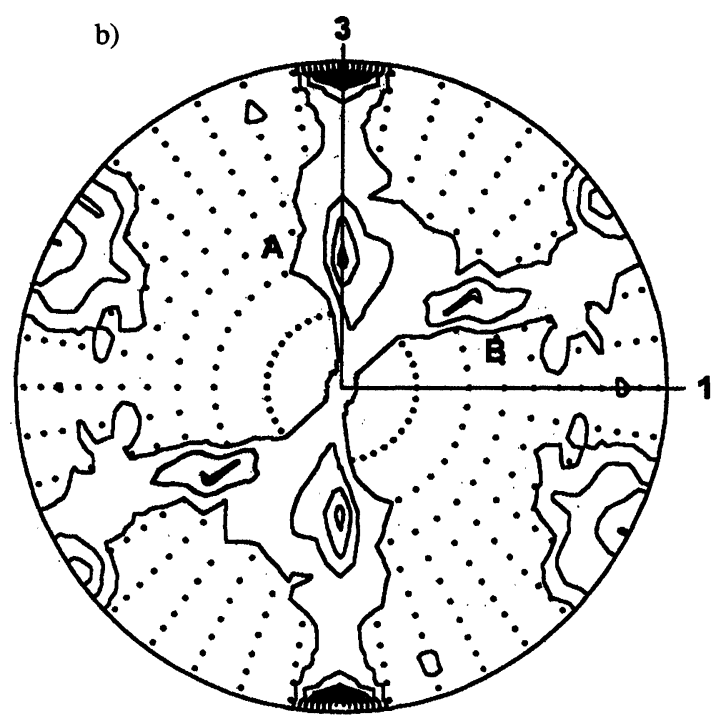

[111] pole figure

Figure 2 a) [111] pole figure for torsion of an FCC material at a Von Mises equivalent strain of 2.00 simulated under Taylor assumption and no limit for the crystal axes spin. Contour levels are multiples of random orientations.

b) Idem under GACM assumptions keeping the crystal axes spin bellow 0.03 per strain step of 0.025 Von Mises equivalent strain. Contour levels are multiples of random orientations. 
is defined as model dependent. Under the current assumptions $\Omega^{\mathbf{D}}$, the spin of the main strain rate axes, is one of the magnitudes to be involved in the definition of the relative spin $\Omega^{\text {rel }}$. It is the action of the applied stress what makes the slip systems glide and it instantaneously defines the main strain rate axes. This assumption has not changed in this model because the response of the material is assumed instantaneous. The other spin to be considered characterizes the spin of the background over which the crystallinity is mounted. In the current model it is characterized by

$$
\Omega_{i j}=w_{i j}-\alpha_{i j} \cdot d_{i j}
$$

and the relative spin becomes:

$$
\Omega_{i j}^{r e l}=\Omega_{i j}-\Omega_{i j}^{D}
$$

The symbols $\Omega$ for the relative spin and the spin of the main strain rate axes mean they are not of the same kind of the antisymmetrical component of the velocity gradient but of the kind that can be written as $\dot{\mathbf{R}} \mathbf{R}^{\mathrm{T}}$. They represent the reorientation of a set of axes and not the average rotation of a bunch of lines that goes through the spinning center.

As in previous charaterization of strain path changes, equation (4.2) states that when $\Omega_{i j}^{\text {rel }}=0$ there will be no strain path change and the texture will become stronger. The lower this parameter the stronger the texture.

\section{CONCLUSIONS}

A new equation has been derived for the grain spin calculation in a polycrystalline material. It has been shown that it is reducible to the Taylor and RC or SC approaches for round and flat grains respectively. A reinterpretation of the strain relaxation concept has also been presented. It allows each grain to compensate the modification of some strain components by keeping the size and shape of the grain in coincidence with the hole ellipsoid. The reorientation of the grain can be calculated if a constitutive relationship is provided to calculate the relaxation. Considering that the reorientation, under the assumptions of previous models, is direct consequence of internal inhomogenities and grain fragmentation and that those mechanisms are energetically unfavorable, a minimum spin principle is suggested. The grain will modify its strain components in such way that the extra spin is the closest to nil that can be compatible with the constraints of the neighborhood. The net behavior of the crystal is a combination of various mechanisms: a) The grain will relax some strain components that will be macroscopically compensated by the deformation of other crystals. This is the behavior usually considered in SC approaches. b) The grain will relax some components that will be internally compensated by the same grain in order to keep, grain by grain, the Von Mises strain in agreement with the average. This is the behavior that is considered in the present paper. c) In order to reorient its crystal axes the grain would inhomogeneously deform and also fragment if no grain boundary debonding is allowed. This kind of behavior would be accessed only by taking in account the local topology of different grains. 


\section{References}

1. Bolmaro, R. E. and Kocks, U. F. (1992). A comparison of the texture development in pure and simple shear and during path changes. Scripta Metall. Mater., 27, 1717-1722.

2. Cauchy, D. (1841). Ex. d'Anal. Phys. Math. 2=Oeuvres (2), 12, 343.

3. Dafalias, Y. F. (1987). Issues on the constitutive formulation at large elastoplastic deformations, Part I: Kinematics. Acta Mechanica, 69, 119-138.

4. Dresselhauss, E. and Tabor M. (1992). The kinematics of stretching and alignment of material elements in general flow fields. J. of Fluid Mech., 236, 415.

5. Guo, Z-H., Lehmann, Th., Haoyun, L. and Man, C. (1992). Twirl tensors and the tensor equation $\mathrm{AX}-\mathrm{XA}=\mathrm{C}$. $J$. of Elasticity, 27, 227-245.

6. Harren, S. V. and Asaro, R. J. (1989). Nonuniform deformationsin polycrystals ans aspects of the validity of the Taylor model. J. Mech. Phys. Solids., 37, 2, 191-232.

7. Kocks, U. F. and Chandra, H. (1982). Slip geometry in partially constrained deformation. Acta Met., 30, 695-709.

8. Kocks, U. F., Dawson, P. R. and Fressengeas, C. (1990). Kinematics of plasticity related to the state and evolution of the material microstructure. Presented at Aristotle Conference on Mechanics, Physics and Structure of Materials in Thessaloniki, Greece, August 1990. To be published in the Journal of Mechanical Behavior of Materials.

9. Kurzydlowski, K. J., Mc. Taggart, K. J., Lazecki, D. and Tangri, K. (1992). The use of grain area measurements to determine the distribution of plastic deformation in polycrystals of austenitic stainless steel. Scripta Met. Mater., 26, 1377-1382.

10. Lebensohn, R. and Bolmaro, R. E. (1993). Comparison of a self-consistent approach and a pure kinematical model for plastic deformation and texture development. To be published ICOTOM 10.

11. Los Alamos Polycrystal Plasticity simulation code: Los Alamos Nat. Lab. LA-CC-88-6 (1988).

12. Mehrabadi, M. M. and Nemat-Nasser, S. (1987). Some basic kinematical relations for finite deformation of continua. Mechanics of Materials, 6, 127-138.

13. Molinari, A., Canova, G. R. and Ahzi, S. (1987). A self consistent approach of the large deformation polycrystal viscoplasticity. Acta Met., 35, 12, 2983-2994.

14. Takeshita. T., Kocks, U. F. and Wenk, H.-R. (1988). The path dependence of deformation texture development. Proc. Eighth International Conference on Texture of Materials. J. S. Kallend and G. Gottstein, eds., TMS, Warrendale, PA. 479-484.

15. Tiem, S., Berveiller, M. and Canova, G. R. (1986). Grain shape effects on the slip system activity and on the lattice rotations. Acta Met., 34, 11, 2139-2149.

16. Zolotorevskiy, N. Yu., Rybin, V. V. and Zhukovskiy, I. M. (1989). Theory of the deformation textures of fragmenting metals. Phys. Met. Metall., 67, 2, 8-18. 